\title{
La Culture Nationale Et L'intégration Relationnelle Des Supply Chains : Etude Qualitative Sur Les Dimensions De La Culture Marocaine Selon Le Modèle De Geert Hofstede
}

\author{
M. Hamri Mohammed Hicham \\ Mme. Zerouali Ouariti Ouafae \\ Mlle. Lechheb Hafsa
}

Ecole Nationale de Commerce et de Gestion d’Agadir, Université Ibn Zohr

doi: 10.19044/esj.2016.v12n35p451 URL:http://dx.doi.org/10.19044/esj.2016.v12n35p451

\begin{abstract}
The relational integration of Supply Chains has provoked a number of rich debates, indicating its importance and relevance for firms seeking performance and gaining significant market share.

However, relational integration of Supply Chains refers to the integration of several management modes, and several national cultures. Therefore, the aim of this paper is to study the dimensions of the Moroccan culture, through a qualitative study, in order to give an answer to this research problem: Does the Moroccan culture present determinants favorable to the relational integration Supply Chains?
\end{abstract}

Keywords : Supply Chain Management, Relational Integration, National Culture, Moroccan Culture.

\section{Résumé}

L'intégration relationnelle des Supply Chains a suscité plusieurs débats riches, indiquant son importance et sa pertinence pour les firmes en quête de la performance, et de gain de parts de marché importantes.

Toutefois, l'intégration relationnelle des Supply Chains désigne l'intégration de plusieurs modes de gestion, et de plusieurs cultures nationales. Par conséquent, le but de ce papier est d'étudier les dimensions de la culture marocaine, à travers une étude qualitative, afin de donner une réponse à cette problématique de recherche: La culture marocaine présente-elle des déterminants favorables à l'intégration relationnelle des Supply Chains ? 
Mots clés : Supply Chain Management, Intégration relationnelle, culture nationale, culture marocaine.

\section{Introduction :}

Une des tendances les plus remarquables sur laquelle il faut mettre l'accent par les entreprises, est la mondialisation. Dans le passé beaucoup d’organisations étaient en compétition avec d'autres entreprises dans leur pays d'origine ou dans leur région. Aujourd'hui, les entreprises énumèrent de multiples partenaires partout dans le monde, s’approvisionnent de la matière première, demandent des prestations, développent des alliances et distribuent leurs produits dans tous les continents.

Désormais les entreprises sont sollicitées à bâtir de puissantes relations avec leurs partenaires basées principalement sur la confiance, et d'intégrer leurs Supply Chains afin d'atteindre parfaitement les objectifs tracés.

L’intégration des Supply Chains représente un point crucial, qui a fait l'objet de plusieurs débats riches (Power, 2005). Au moment où les firmes cherchent à construire des liens avec leurs partenaires, les processus internes sont interconnectés, et s’étendent sur les frontières des entreprises (Power, 2005).

Par conséquent, l’intégration des Supply Chains demeure donc un point vital, pour les firmes qui cherchent à atteindre un avantage concurrentiel et gagner des parts de marché, dans un monde marqué par une concurrence féroce.

Par ailleurs, l'intégration des Supply Chains offre une multitude d’opportunités pour les organisations, toutefois, ces opportunités ne viennent pas sans éventuelles entraves. La confiance, notamment, représente une condition préalable au succès de l'intégration des Supply Chains, mais la confiance et sa pertinence peuvent changer d'une culture nationale à une autre.

Dans le présent papier, nous allons présenter notre problématique de recherche, étudier les fondements théoriques de la culture nationale et de l'intégration des Supply Chains, et analyser les résultats de notre étude qualitative exploratoire sur les spécificités de la culture marocaine, en nous focalisant, plus particulièrement, sur le modèle fondateur de la culture nationale de Geert Hofstede.

\section{Problématique de recherche :}

Le Maroc représente un pays qui a un potentiel énorme d’amélioration de la compétitivité de son économie à l’instar d'une logistique considérable optimisant et ordonnant tous les flux de marchandises. De ce fait le Maroc a mis en œuvre la Stratégie Nationale de Développement de la Compétitivité Logistique qui va dégager des réponses cruciales pour le rayonnement de la 
logistique et qui va inscrire le Royaume dans les Supply Chains les plus performantes.

L’inscription des entreprises marocaines dans les Supply Chains, exige un changement dans les outils et méthodes de gestion et de partage de l'information, qui peut faire l'objet de plusieurs feed-back positifs ou négatifs de la part des responsables et des employés. L'acceptation ou le refus est inhérent à un certain nombre de paramètres relatifs à la culture nationale. Comme l'explique Philippe d'Iribarne, que la culture est un système de sens à travers lequel l'individu perçoit est interprète une situation ou une action concrète, ce système de sens est partagé avec les autres membres de sa communauté, qu'il a au cours de son histoire, constitué.

La prise en compte de la culture nationale dans l'intégration relationnelle des Supply Chains, demeure un point central à évoquer lors de l'adoption de ce nouveau mode de gestion, afin de garantir au maximum son succès.

Ce travail de recherche a pour but d'explorer les déterminants de la culture marocaine et d'analyser leur influence et leur impact sur l'intégration des Supply Chains, en répondant à la question suivante: La culture marocaine présente-elle des déterminants favorables à l'intégration relationnelle des Supply Chains?

\section{Cadre conceptuel de la culture nationale et de l'intégration relationnelle des Supply Chains :}

Dans la présente partie nous allons présenter les fondements théoriques de la culture nationale, en se basant plus particulièrement sur le modèle fondateur de la culture nationale de Geert Hofstede, ainsi que les fondements théoriques de l'intégration des Supply Chains, en argumentant notre choix de l’intégration relationnelle des Supply Chains comme objet de notre étude.

\subsection{Culture nationale :}

Nous pouvons définir un pays comme un territoire composé d’individus qui représente une autorité politique, établie sur un espace géographique défini et incarnée par une autorité souveraine ${ }^{76}$. L'idée de nation suppose une construction historique et donc un passé. Elle s’exprime également dans le présent, par la volonté clairement exprimée par les citoyens de poursuivre leur vie en commun, suivant les règles dictées par la société (langue commune, pratiques religieuses, coutumes du pays... $)^{77}$. La culture nationale désigne alors l'ensemble des valeurs et des traits distinctifs d'un groupe d’individus appartenant au même pays.

\footnotetext{
${ }^{76}$ Définition issue du Dictionnaire Petit Robert, complétée par les écrits de E. Renan, Qu'est ce qu'une nation? INALF, 1961.

${ }^{77}$ Oliver Meier, Management interculturel, Stratégie, Organisation, Performance, Dunod, Paris, 2008, p.22.
} 
Afin de traiter les déterminants de la culture nationale, notre avons opté pour le modèle de Geert Hofstede, bien que divers modèles culturels étaient proposés dans la littérature, et malgré beaucoup de critiques (Mc Sweeny, 2002), l'applicabilité du modèle d'Hofstede dans les travaux inscrits en sciences de gestion, et sa validité, comparé à d'autres modèles culturels, demeurent remarquables (Merrit, 2000, Magnusson et al, 2008). Le modèle d'Hofstede est adopté également dans les travaux qui ont pour objectif la comparaison entre les cultures nationales (Magnusson et al, 2008, Merrit, 2000). Finalement le modèle d'Hofstede représente le modèle fondateur de la culture nationale, et montre une certaine facilité de compréhension et une explication claire des déterminants de la culture nationale.

Le modèle de Geert Hofstede est composé de cinq principaux déterminants de la culture nationale :

\section{$>$ La distance hiérarchique :}

La distance hiérarchique est définie par Hofstde (1980) comme la perception du degré d'inégalité du pouvoir entre un subordonné et son supérieur. Cette dimension fait référence au degré d'inégalité attendu et accepté par les individus. Dans les sociétés à forte distance hiérarchique, le pouvoir est centralisé et le leadership est exercé de manière autocratique et coercitive (Hofstede, 1983). Contrairement aux pays où la distance hiérarchique est faible, l'accent est mis sur l'égalité et sur la coopération.

\section{$>$ L'orientation individualiste ou communautaire :}

Selon Hofstede, la distinction entre les cultures nationales peut se faire également à travers les relations que les individus entretiennent avec les autres membres de la collectivité.

Cette dimension renvoie à l'importance donnée par un individu à sa propre personne ou à son entourage (Hofstede, 1983,1991). Dans les sociétés marquées par l'individualisme, les individus se préoccupent davantage de leurs propres intérêts et de leur environnement immédiat (Hofstede, 1994). Alors que dans les pays à culture communautaire, l'importance est donnée aux liens affectifs et aux relations de confiance unissant les membres de la communauté.

\section{La relation à l'incertitude et à l'ambigüité :}

Cette troisième dimension renvoie au degré de tolérance de l'incertitude et de l'ambigüité. Le niveau de contrôle de l'incertitude signifie le degré jusqu'où les individus d'une culture acceptent les situations ambigües, risquées, imprévisibles, non-structurées, voire anarchiques et la manière avec laquelle ces membres réagissent à cette imprévisibilité (Hofstede, 1983,1991). 
Le contrôle de l'incertitude peut être mis en relief à travers des plans et des outils de prévision, de recherche de stabilité et de l'ordre. Le faible degré de contrôle de l'incertitude se traduit par la prise de risque, et la confrontation des événements futurs ambigus.

\section{L'orientation masculine ou féminine des valeurs :}

Les cultures masculines accordent une grande importance aux valeurs de la réussite et de la possession, alors que les cultures féminines mettent l'accent surtout sur les valeurs de l'entraide et de l'empathie, dans un souci de cohésion sociale.

\section{L'orientation à court terme et l'orientation à long terme :}

Les cultures à orientation à court terme, sont des cultures qui respectent la tradition, l'exécution des obligations sociales, et considèrent que le service d'autrui est un but important. Ces cultures ont comme valeurs principales du travail ; la liberté, le respect des droits, la réussite et l'épanouissement personnel.

Les cultures à orientation à long terme encouragent la persévérance, la prévoyance et le sens de l'épargne. Ces cultures ont comme fondamentales valeurs du travail ; l'apprentissage, l'honnêteté, la responsabilité et l'autodiscipline.

Vu que nous étudions les déterminants de la culture marocaine, et pour respecter et refléter au maximum la réalité de notre contexte de recherche, nous allons ajouter un autre déterminant même s'il ne figure pas dans le modèle d'Hofstede, celui de la religiosité.

En effet, malgré que la religiosité ne figure pas parmi les déterminants de la culture nationale proposés par Hofstede, nous confortons notre choix par des travaux antérieurs (Hofstede, 1991, Schwartz et Huismans, 1995, Saroglou et al, 2004, El Akremi, 2007, Balambo, 2013, 2014), qui ont intégré la religiosité, afin d'appréhender le plus quelques sociétés caractérisées par la religion, cette dernière qui peut jouer le rôle d'un facteur explicatif des attitudes, des comportements et des relations avec autrui.

La religiosité peut être définie comme le niveau de participation, ou de fidélité aux convictions ou aux pratiques d'une religion (Shaffer, 1996). La religiosité est vue comme l'ensemble des attitudes et des pratiques pour découvrir le sens, le but ou la connexion avec des choses externes et plus puissantes que le Moi (Kieshing et al, 2008).

\subsection{Intégration des Supply Chains :}

Le concept d'intégration des Supply Chains est un élément central du Supply Chain Management (Baharanchi, 2009; Danes et al, 2013), en effet, 
l’intégration est fréquemment notée comme une clé caractéristique du Supply Chain Management (Dag Naslund Hana Hulthen, 2012).

Plusieurs définitions du Supply Chain Management se rapportent à l'intégration (Pagell, 2004 ; Stock et Boyer, 2009), notamment celle de Cooper et al (1997) qui considère le Supply Chain Management comme une philosophie qui tend vers une gestion intégrée de l'ensemble des flux d'un canal de distribution, du fournisseur à l'utilisateur final.

Mentzer et al (2001) étudient comment l'intégration représente un élément essentiel pour l'implantation du Supply Chain Management, tandis que Lambert et al (1998) affirment que le but de l'intégration est d'améliorer l'efficacité et l'efficience de tous les maillons de la Supply Chain.

Par ailleurs, l'intégration des Supply Chains peut être identifiée en six types, il convient de noter, l'intégration stratégique, l'intégration des relations, l'intégration interne, l'intégration externe, l'intégration de l'information et l'intégration des mesures (Baofeng et al, 2005).

L'intégration stratégique se réfère au degré auquel une entreprise peut structurer les buts et les objectifs stratégiques, ainsi que le partage des ressources, des récompenses et des risques entre les organisations, à travers un consensus et des accords contractuels afin d'atteindre la compétitivité (Burgelman et Doz, 2001 ; Fuchs et al, 2000 ; Johnson, 1999).

L'intégration des relations est définie comme le degré auquel une entreprise peut structurer la formation, l'engagement, l'entretien et la sortie des relations entre les organisations, à travers un consensus et des accords contractuels afin d'atteindre la compétitivité (Bowersox et al, 1999 ; Stank et al, 2001).

L'intégration interne se refère au degré auquel une entreprise peut structurer ses pratiques, ses procédures et ses comportements organisationnels en processus collaboratifs, synchronisés et gérables afin de répondre aux exigences des clients (Cespedes, 1996 ; Chen et Paulraj, 2004 ; Kahn et Mentzer, 1996 ; Kingman-Brundage et al, 1995).

L’intégration externe est considérée comme le degré auquel une entreprise peut s'associer avec ses principaux membres de la Supply Chain, pour structurer leurs pratiques, procédures et comportements interorganisationnels en processus collaboratifs, synchronisés et gérables afin de satisfaire les exigences des clients (Stank et al, 2001).

L'intégration de l'information désigne le degré auquel une entreprise peut coordonner les activités de partage de l'information, par le biais de plates formes communes (Jhingran et al, 2002 ; Roth et al, 2002).

Finalement, l'intégration des mesures qui est définie comme le degré auquel une entreprise peut structurer les systèmes de mesures, et gérer les activités de mesure avec ses principaux membres de la Supply Chain, afin de 
concrétiser la stratégie d’intégration des Supply Chains (Bowersox et al, 1999).

New (2004) quant à lui, affirme que l’intégration des Supply Chains peut être comprise de trois façons, à savoir l'intégration opérationnelle (coordination des inventaires, ordonnancement, transport, développement de nouveaux produits), l'intégration fonctionnelle (gestion des différentes fonctions managériales telles que l'achat et la gestion des stocks), et l’intégration relationnelle (amélioration des relations avec les partenaires).

\subsection{Choix de l'intégration relationnelle des Supply Chains :}

Dans ce papier, nous allons nous positionner sur l'aspect relationnel de l'intégration des Supply Chains, autrement dit l'intégration des relations, ou encore l'intégration comportementale (Bowersox et al, 1996, 1999, 2000 ; Mentzer et al, 2001), car cette dernière constitue la pierre angulaire du Supply Chain Management (Mentzer et al, 2001).

Nous remarquons à travers notre revue de littérature, que la majorité des travaux de recherche se concentrent essentiellement sur les aspects opérationnels et technologiques, servant à la mise en place d'une intégration efficace des maillons de la Supply Chain, d'où le choix de notre positionnement sur le côté relationnel de l’intégration des Supply Chains.

L’intégration relationnelle est considérée comme la capacité d'une entreprise à s'appuyer sur la confiance, l'engagement, l'entretien de la relation, voire même le consensus en cas de rupture d'une relation (Bowersox et al, 1999).

L’intégration relationnelle s’appuie sur un processus partenarial (Cooper et al, 1997), qui se base sur de nouveaux principes d'interprétation des relations inter-organisationnelles, ces relations qui exigent une réelle concordance, voire une congruence culturelle (Lassar et Zinn, 1995 ; Mentzer et al, 2001 ; Baratt, 2004 ; Adam-Ledunois et Renault, 2004).

\section{Présentation des résultats de l'étude exploratoire sur les spécificités de la culture marocaine :}

Nous avons constaté à travers notre revue de littérature, qu'il existe peu de travaux qui ont traité le contexte marocain et ses particularités selon le modèle de Geert Hofstede. Par conséquent, afin d'enrichir et de compléter davantage les conclusions tirées de la revue des travaux précédents, nous allons présenter, dans la présente partie, les résultats de notre étude exploratoire basée sur une démarche abductive, sur les spécificités de la culture marocaine, après avoir précisé les éléments méthodologiques utilisés. 


\subsection{Eléments méthodologiques utilisés :}

Notre étude qualitative exploratoire s'est effectuée à travers des entretiens individuels semi-directifs, en se basant sur des guides d'entretien. Ces entretiens se sont déroulés avec 10 professeurs en sociologie et en anthropologie de diverses universités marocaines, à savoir l’Université Mohamed V de Rabat, l’Université Ibn Tofail de Kenitra, et l’Université Ibn Zohr d'Agadir.

Dans le but de fixer la taille de notre échantillon, nous nous sommes basés sur le principe de saturation de l'information fourni par Glaser et Strauss (1967).

Par ailleurs, nous avons opté pour la méthode résumé/synthèse, qui se fonde sur deux approches complémentaires d'analyse des entretiens, qui sont l'approche verticale et l'approche horizontale.

\subsection{Résultats de l'étude exploratoire :}

Dans la présente partie nous allons présenter les résultats de notre étude exploratoire sur les déterminants de la culture marocaine, en se basant sur le modèle de Geert Hofstede.

L’analyse des résultats de notre étude qualitative s'est effectuée à travers la confrontation des conclusions de nos répondants, et la revue de littérature sur les spécificités de la culture marocaine.

\subsubsection{Culture marocaine et distance hiérarchique :}

Notre revue de littérature sur les spécificités de la culture marocaine, nous a révélé que la culture marocaine est marquée par le principe de la hiérarchie prononcée et par le principe d'autorité (Bourqia, 2010).

De la même manière, d'autres études ont montré le fort degré hiérarchique existant au Maroc (Mezouar et Semeriva, 1998 ; Al Maache, 2002 ; Eddakir, 2003, Nouiga, 2003 ; House et al, 2004 ; Balambo, 2014).

La distance hiérarchique et les inégalités sont attendues et acceptées par les marocains, et sont traduites généralement par la notion de "arzaq » et " aqdar », comme l'un de nos répondants a affirmé que " généralement les inégalités sont interprétées de point de vue religieux en mettant en avant la notion de "arzaq" ou "aqdar». Les inégalités ne sont pas dues à des politiques ou à des conditions historiques et idéologiques, mais au "mektoub» qui veut dire que les conditions de vie et la situation dans laquelle vit chaque être humain sont décidée bien avant sa naissance ».

Par conséquent, ces conclusions coïncident avec les résultats de notre étude exploratoire, qui ont affirmé que la culture marocaine présente un fort degré hiérarchique. 


\subsubsection{Culture marocaine et orientation individualiste ou communautaire :}

A travers notre revue de littérature sur les spécificités de la culture marocaine, nous avons constaté qu’elle est caractérisée par un niveau élevé de collectivisme (Hofstede, 1980, 2001; Mezouar et Semeriva, 1998 ; Al Maache, 2002 ; Eddakir, 2003; Nouiga, 2003 ; House et al, 2004 ; Matsumoto, 2006 ; Hmaida, 2010, Allali, 2010 ; Balambo, 2014). De même, ces conclusions sont en concordance avec les résultats de notre étude exploratoire.

L'un de nos répondants a affirmé que «la culture marocaine est une culture à dominance collectiviste, qui accorde une importance indéniable aux relations sociales. Des relations qui se voient considérées comme étant un gisement où tout marocain puise ses repères de sociabilité, d'identité et d'appartenance ».

\subsubsection{Culture marocaine et orientation masculine ou féminine des valeurs :}

Notre revue de littérature a démontré que la culture marocaine a tendance à adopter une orientation féminine des valeurs (Nouiga, 2003 ; Bourqia, 2010 ; Balambo, 2014). En effet, notre étude exploratoire a généré des résultats semblables aux constats précédents.

Geert Hofstede (2010) a annoncé également que les sociétés marquées par une orientation féminine des valeurs, sont caractérisées par l'humanisation du travail, par le contact et la coopération, par l'entraide et la collaboration dans le cadre des relations sociales également.

Par conséquent, comme l'un de nos répondants a indiqué que « en tant que culture marquée par la diversité et le pluralisme, la culture marocaine est riche en valeurs « tendres » de solidarité, de tolérance, d'unité, d'importance des relations sociales, de coopération et d'ouverture.

\subsubsection{Culture marocaine et relation à l'incertitude et à l'ambigüité :}

A partir de notre revue de littérature, nous avons découvert que plusieurs études intéressées au contexte marocain, ont montré que la culture marocaine est caractérisée par un faible degré du contrôle de l'incertitude (Nouiga, 2003 ; Eddakir, 2003 ; Balambo, 2014). Ce faible niveau de contrôle de l'incertitude se ressource de notre religion et de la notion du Maktoub (Al Maache, 2003).

En effet, notre étude exploratoire a révélé que le risque et l'incertitude sont interprétés par Lmektab et la volonté de Dieu. Comme nous a affirmé l'un de nos répondants que "c'est Lmektab qui interprète ce genre de situation dans notre société ». Conjointement, Geert Hofstede (2010), a précisé que dans les cultures à faible contrôle de l'incertitude, le risque et 
l'ambigüité font partie de la vie de chaque jour, et acceptés pour ce qu'ils sont. "Selon notre culture, il faut se remettre au destin », comme nous indiqué l'un de nos répondants.

\subsubsection{Culture marocaine et orientation à court terme ou à long terme :}

A travers notre revue de littérature, nous avons conclu que la culture marocaine est spécifique par une orientation à court terme, qui se traduit par un attachement fort de la culture marocaine, aux traditions et aux normes sociales. Comme a affirmé Bourqia (2010), que la coutume, la tradition, les droits coutumiers sont les principales sources de valeurs, véritables référentiels de valeurs morales, qui régissent l'appréciation de l'individu, les relations sociales et les rapports à la communauté.

En effet, les propos de l'un de nos répondants ont montré que " malgré les mutations que connait la société marocaine, il me semble que les normes sociales les traditions ont encore leur place et jouent un rôle important dans les relations sociales entre individus dans la vie privée et la vie professionnelle ».

D’après Geert Hofstede (2010), les cultures à orientation à court terme sont toujours liées au passé. La culture marocaine a, justement, une projection dans l'avenir, mais elle reste marquée par le passé, comme nous a expliqué l'un de nos répondants.

\subsubsection{Culture marocaine et religiosité :}

A travers notre revue de littérature, nous avons conclu que la culture marocaine, est une culture à forte religiosité (Geertz, 1973 ; d'Iribarne, 1997 ; Al Maache, 2002 ; Bourqia et al, 2007 ; Tozy et al, 2007 ; Bourqia, 2010 ; Balambo, 2014).

En effet, l'un de nos répondants nous a indiqué que " à mon sens, la religion peut être considérée comme pierre angulaire et composante principale de la culture marocaine, elle revêt une importance très significative dans la vie des marocains, et leur vision des choses, et constitue ainsi une source d'inspiration majeure des codes sociaux, un cadre de référence par excellence, et un véritable réservoir des valeurs éthiques et morales ».

\section{Conclusion :}

Dans le présent papier, nous nous sommes intéressés à l'exploration des spécificités de la culture marocaine selon le modèle de Geert Hofstede, vu qu'il existe peu de travaux de recherche de ce type, à travers une étude qualitative exploratoire qui s'est effectuée à traves des entretiens individuels et semi directifs, avec des professeurs universitaires en sociologie et en anthropologie. 
Cette étude exploratoire nous a révélé que la culture marocaine présente les caractéristiques suivantes :

- Une forte distance hiérarchique ;

- Une orientation communautaire ;

- Un degré faible du contrôle de l’incertitude ;

- Une orientation féminine des valeurs ;

- Une orientation à court terme ;

- Une forte religiosité.

Les résultats de cette étude qualitative vont nous aider à mener une autre étude quantitative confirmatoire, à travers laquelle nous allons essayer de répondre à notre problématique de recherche.

\section{References:}

1. Allali Brahim. (2008), Culture et gestion au Maroc: une osmose atypique, dans Eduardo Davel, Jean-Pierre Dupuis et Jean-François Chanlat (dir.), Gestion en contexte interculturel: approches, problématiques, pratiques et plongées, Québec, Presses de Laval et Télé-université (UQAM).

2. Ali A. J., Wahabi R. (1995), Managerial value systems in Morocco, International Studies of Management \& Organization, vol. 25, n³, p. 87-96.

3. Azzedine TOUNÉS, Khalil ASSALA. (2007), Influences culturelles sur des comportements managériaux d'entrepreneurs algériens, 5ème congrès de l'Académie de l’Entrepreneuriat Sherbrooke - Canada.

4. Balambo M. A. (2013), Culture nationale et nature de l'intégration des supply chains amont: le cas des équipementiers automobiles marocains, Logistique \& Management, vol. 21, n4 , p. 71-85.

5. Balambo M. A. (2014), Hofstede's model revisited: an application for measuring the Moroccan national culture, International Journal of Business Quantitative Economics and Applied Management Research, Vol.1, n³.

6. Baofeng Huo, Xiande Zhao, Jeff Hoi Yang Yeung, Power (2005), Relationship Commitment and Supply Chain Integration Between Manufacturer and Supplier, Proceeding of the fifth International Conference on Electronic Business, Hong Kong, December 5-9, p.868-881.

7. Barratt M. (2004), Understanding the meaning of collaboration in the supply chain. Supply Chain Management: an international journal, vol.9, $\mathrm{n}^{\circ} 1$, p.30-42. 
8. Bourquia R., (2006), « Les valeurs Changements et perspectives », Valeurs et Développement Humain.

9. Doney P. M., Cannon J. P., Mullen M. R. (1998), Understanding the influence of national culture on the development of trust, Academy of management review, Vol.23, n³, p.601-620.

10. El Akremi, A., Ikram Nasr, M., \& Sassi, N. (2007), Impact de la culture nationale sur la confiance interpersonnelle en milieu du travail: analyse comparative entre la France et la Tunisie, Les actes des congrès AGRH.

11. Feng W. (2010), Le supply chain management et la culture : la mise en oeuvre du Supply Chain Management dans le contexte culturel chinois, Thèse de Doctorat en Sciences de Gestion, Université d'AixMarseille II, France.

12. Gavard-Perret M, Gotteland D, Haon C \& Jolibert (2008), Méthodologie de la recherche, Réussir son mémoire ou sa thèse en sciences de gestion, Pearson Education, France.

13. Haider I. (2014), Collaboration in Supply Chain Management: What it is and How to use it? (An intenational review), International Journal of Research, Vol.1, n6, p.499-507.

14. Hofstede G. (1993), Cultural constraints in management theories, The Academy of Management Executive, vol.7, $\mathrm{n}^{\circ} 1, \mathrm{P} .81-94$.

15. Hofstede G, Hofstede G.J \& Minkov M. (2010), Cultures et organisations, Comprendre nos programmations mentales, $3^{\text {ème }}$ édition, Pearson Education.

16. Hofstede G. (2011), Dimensionalizing cultures: The Hofstede model in context, Online readings in psychology and culture, vol.2, n¹, P.8.

17. Hofstede G. (1983), The cultural relativity of organizational practices and theories, Journal of international business studies, Vol.14, $\mathrm{n}^{\circ} 2$, P.75-89.

18. Hofstede G. (1998), Think locally, Act globally: Cultural Constraints in Personnel Management, Management International Review, Vol.38, Cross Cultural and Comparative International Human Ressource Management, p.7-26.

19. Mentzer J. T., DeWitt W., Keebler J. S., Min S., Nix N. W., Smith C. D., Zacharia Z. G. (2001). Defining supply chain management, Journal of Business Logistics, Vol.22, n², p.1-25.

20. Power, D. (2005), Supply chain management integration and implementation: a literature review, Supply chain management: an International journal, vol.10, n4, P. 252-263.

21. Schwartz S. H., Huismans S. (1995), Value priorities and religiosity in four western religions, Social Psychology Quterly, vol.58, n², p.88107. 
22. Stank T. P., Keller S. B., Closs D. J. (2001), Performance benefits of supply chain logistical integration, Transportation Journal, vol.41, $n^{\circ} 2 / 3$, p. 32-46.

23. Yvonne M., Everdingen V., Waarts E. (2003), The effect of National Culture on the adoption of innovations, Marketing Letters, Vol.14, $\mathrm{n}^{\circ} 3$, p. 217-232. 